\title{
Niñas, hasta que un niño diga lo contrario: lenguaje y presencia
}

\author{
CATALINA IZQUIERDO* \\ Universidad de Cantabria - España
}

SANTIAGO ROSANO**

Universidad de Cuenca - Ecuador

\section{RESUMEN}

Este trabajo es una reflexión sobre la relación entre el lenguaje y la presencia. Se cuestionan las directrices de la Academia Española, que defiende el uso del masculino para referirse a ambos sexos. Da la impresión que ser niña es posible mientras no hay un niño presente, pues entonces se invisibiliza su ser femenino. Las normas de la Academia, hechas desde una visión del lenguaje meramente lingüística, son refutadas por autoras que tienen en cuenta la carga ideológica del mismo.

Se presenta información de una sociedad todavía machista. Se defiende un lenguaje que visibilice ambos sexos, para aportar al fortalecimiento de una cultura que los valore de la misma forma; y si se opta por un género en el lenguaje hacerlo por el que desvele al sexo marginado.

Palabras clave: niñas, lenguaje, machismo, visibilizar, femenino.

Girls, until a boy says the opposite: language and presence

\section{Abstract}

This work is a reflection on the relationship between the use of language and presence. The guidelines given by the Royal Spanish Academy, which advocates the use of male generically, are questioned. It seems that being a girl is possible

\footnotetext{
Máster en educación parvularia, en 2008, en la Universidad de Cuenca, Ecuador; Máster en investigación e innovación en contextos educativos, en la Universidad de Cantabria, España. Ha trabajado como profesora de la carrera de Educación General Básica de la Universidad de Cuenca, Ecuador. Doctoranda por la Universidad de Cantabria, España. Contacto: anitacatalina.izquierdo@alumnos.unican.es

** Profesor de la carrera de Educación General Básica de la Universidad de Cuenca, Ecuador. Doctor por la Universidad de Cantabria, España. Contacto: santiago.rosano@ucuenca.edu.ec
} 
as long as there is not a boy present, at that time her feminine identity is invisible. The rules of the Royal Academy, made from a vision of purely linguistic language, are refuted by several authors who argue that the language has a strong ideological charge.

The article gives information about our sexist society, for this reason it defends a language who nomine both sexes, and thus contribute to a culture that values them in the same way; and, therefore, if you opt for a genre in the language, made visible the sex that is marginalized.

Key words: children, languaje, machismo, make visible, female.

\section{Meninas, até que um menino diga o contrário: linguagem e presença}

\section{Resumo}

Este trabalho é uma reflexão sobre a relação entre linguagem e presença. Questiona-se as diretrizes da Real Academia Espanhola que defende o uso do gênero masculino para referir-se a ambos os sexos. Parece que "ser menina" somente é possível enquanto não há um menino presente, pois a presença masculina torna invisível o ser feminino. As normas da Real Academia Espanhola, estruturadas a partir de uma visão meramente linguística, são refutadas por várias autoras que defendem a importância de levar em consideraçáo a carga ideológica das mesmas.

As informaçóes apresentadas demonstram uma sociedade ainda dominada pelo machismo. Portanto, defende-se uma linguagem que mencione ambos os sexos, fomentando assim uma cultura que os valorize da mesma forma. Neste sentido, e em caso de optar por apenas um dos gêneros, dever-se-ia dar mais visibilidade ao sexo marginalizado.

Palavras-chave: meninas, linguagem, machismo, visibilidade, feminino. 


\section{LO MASCULINO COMO GENÉRICO}

Un objetivo manifiesto del Ministerio de Educación [de Ecuador] es combatir el sexismo y la discriminación de género (...) y promover, (...) la equidad entre mujeres y hombres. Para alcanzar este objetivo, promovemos el uso de un lenguaje que no reproduzca esquemas sexistas, y de conformidad con esta práctica preferimos emplear (...) palabras neutras, tales como las personas (en lugar de los hombres) o el profesorado (en lugar de los profesores). Solo en los casos en que tales expresiones no existan, se usará la forma masculina como genérica para hacer referencia tanto a las personas del sexo femenino como masculino (...) para así evitar el abultamiento gráfico y la consiguiente ilegibilidad que ocurriría en el caso de utilizar expresiones como las y los, os/as y otras fórmulas que buscan visibilizar la presencia de ambos sexos (Ministerio de Educación, 2014, p. 4).

Es común encontrar al inicio de los libros o artículos notas aclaratorias explicando la postura que la o las autoras han tomado en el uso del lenguaje respecto a las palabras con género femenino o masculino; y habitualmente la opción es por el masculino. El anterior párrafo, utilizado por el Ministerio de Educación de Ecuador, es un ejemplo. Elegimos este porque, si bien no compartimos la idea, es de los más sensatos que conocemos pues demuestra una actitud de preocupación por el tema.

A pesar de los esfuerzos y avances habidos, los hechos muestran que nuestra sociedad sigue discriminando a la mujer. Mayormente son los hombres quienes ocupan los principales puestos de poder en los gobiernos y empresas. El Informe de Desarrollo Humano 2015 de Naciones Unidas seńala que a nivel mundial las mujeres ocupan el $22 \%$ de los escaños de los parlamentos nacionales (Público, 2015). En la Asamblea ecuatoriana el porcentaje, si bien sigue mostrando el poder masculino, es menos desigual: las mujeres alcanzan el $43 \%$. El informe referido de Naciones Unidas indica que en el mundo empresarial las mujeres solo ocupan el $25 \%$ de los cargos administrativos y directivos, pese a que su contribución al trabajo mundial es del 52\% (Público, 2015).

En educación el panorama no varía, veamos dos ejemplos cercanos a quienes escribimos. De las doce facultades que hay en la universidad de Cuenca (Ecuador) tres están dirigidas por mujeres y nueve por hombres. En la investigación realizada en dos escuelas de Cuenca (Rosano, 2015) ${ }^{1}$, curiosamente las 28 maestras responsables de aula de primero a séptimo eran mujeres, mientras que quien ejercía la dirección, en ambas escuelas, era hombre.

\footnotetext{
1 El tema no estaba, en principio, relacionado con el género, trataba la participación estudiantil.
} 
El aspecto salarial es quizá uno de los que con más crudeza muestra cuánto "valen menos" las mujeres: a nivel mundial, según señala Naciones Unidas, las mujeres ganan un 24\% menos que los hombres (Público, 2015). Veamos de nuevo el ejemplo de un país "occidental” y "avanzado" como Alemania: allí el salario promedio de las mujeres es un $22 \%$ menor que el de los hombres por trabajos similares (ABC, 2014). Eso quiere decir que para tener los mismos ingresos que los hombres deberían trabajar al año 82 días más. En Ecuador la situación se muestra parecida: un 26\% menor (INEC, 2012).

La violencia de género que sufren las mujeres es otro indicador vergonzoso de nuestra sociedad machista. En Ecuador, 6 de cada 10 mujeres declaran haber vivido violencia de género (Camacho, 2014, p. 32). Y si miramos de nuevo hacia Europa, encontramos casos igualmente preocupantes: en España, en 2015, al menos 57 mujeres fueron asesinadas por los hombres con los que tenían o habían mantenido una relación de pareja ese ańo (El Mundo, 2016).

\section{VISIBILIZAR LO FEMENINO}

Y, ¿qué tienen que ver estos hechos con el lenguaje del que hemos empezado hablando? El que llevemos milenios sobrevalorando al hombre en perjuicio de la mujer y que en el lenguaje impere el masculino no son hechos inconexos; el vínculo palabra-acción es fuerte. Desde diferentes ámbitos de estudio se ha abordado esta relación. De una forma muy directa, Eulàlia Lledó (2004) estudia la necesidad de nombrar a las mujeres para describir la realidad. Jerome Bruner (1989) aborda la relación entre el lenguaje y la cultura: el lenguaje crea cultura, crea realidad, y yendo al campo escolar dice que se deberían analizar las consecuencias de nuestra forma de hablar en la escuela de manera que nadie sea marginada. Esta relación la estudia también Marta Lamas (2007): el lenguaje, como elemento fundamental de la cultura, actúa en el desarrollo cognoscitivo de las niñas y niños de modo que asumen diferencias entre unas y otros, antes incluso de tener claras las diferencias de sexo. José Flores y Anna Holzscheiter (2008) analizan la relación entre el poder y el discurso, así como Carlos Lomas (2011) lo hace entre las palabras y el poder, como ambos se formular y reformulan. Lev Vigotsky (1995) estudió la relación entre el pensamiento y el lenguaje. Una de sus más importantes aportaciones fue explicar la construcción del mundo cognitivo y afectivo humano ${ }^{2}$ como resultado de la comunicación, es decir, de la creación y uso de signos. Dicho de otra forma:

\footnotetext{
2 Estos componentes cognitivo y afectivo forman la conciencia humana: objeto de estudio de Vigotsky para comprender el comportamiento humano (Vila, 2009, p. 216).
} 
el lenguaje genera pensamiento y este, a su vez, lenguaje (Myers, 2006). Y concretando en el tema que nos ocupa, decimos que la palabra actúa "en el mantenimiento o la construcción de estereotipos y referentes sexuales que pueden suponer un freno a los avances sobre igualdad sexual o, por el contrario, estimular el cambio" (Universidad de Cantabria, 2011, p. 6). La palabra actúa, la palabra tiene mucho que decir.

No obstante, la Real Academia Española de la lengua, paladín del lenguaje, afanada y absorta en limpiar, fijar y darle esplendor, se posiciona en favor de su neutralidad. Y repite por activa y por pasiva, en presente y en futuro, en infinitivo y en imperativo, que mencionar, por ejemplo, los niños y las niñas, es un desdoblamiento artificioso e innecesario (RAE, 2016b).

De todas formas, lo que dictamine la Real Academia en este tema lo vemos con recelo, siendo una institución con significativas prácticas que marginan a la mujer. En sus 300 años de vida, ha habido 11 mujeres frente a centenares de hombres. En la actualidad, de las 45 personas que la componen, 8 son mujeres. Por supuesto, ninguna de las 30 personas que la han dirigido, a lo largo de su historia, ha sido mujer (RAE, 2016a). Una de las pistas para entender estas prácticas nos la da precisamente una de las mujeres que componen esta institución, Clara Janés, quien afirma: "no me he planteado nunca si la RAE es machista o no" (Remacha, 2016). Quizá, Señora Clara, justamente ese sea el problema.

Nuria Pérez de Lara explica claramente cómo la reducción de los dos sexos a uno en el lenguaje responde, bajo el aparente pretexto de la simplificación, a una subordinación de la otra persona, en este caso la femenina.

No considerar la diferencia sexual puede entenderse como una especie de decisión simplificadora. Pero, si profundizamos, podremos ver que (...) aquí lo que se excluye es la alteridad misma por la que se constituye el sujeto humano a causa del sexo (...). En esto ha jugado un papel importante el dominio sexista. La subordinación de un sexo al otro es una manera práctica de resolver el problema del sujeto humano que no es uno sino dos (Diotima, 2002, citada en Pérez de Lara, 2010, p. 126).

No es igual referirnos a los niños que hacerlo a las niñas y niños. En el primer caso, en aras de la "ley lingüística de la economía expresiva" ${ }^{3}$ o simplemente de la tradición, inconscientemente estaremos contribuyendo a mantener la situación actual: donde se relega lo femenino hasta la anulación; estaremos

3 Expresión utilizada por la Real Academia Española para referirse a que "en la lengua está prevista la posibilidad de referirse a colectivos mixtos a través del género gramatical masculino" (RAE, 2005). 
alimentando la idea de que el lenguaje masculino incluye al femenino (que es lo mismo que decir que el mundo masculino incluye al femenino). En el segundo caso (referirnos a las niñas y niños), teniendo en cuenta que el lenguaje "es una convención social que ayuda a conformar la realidad y nos enseña a ver el mundo" (Universidad de Cantabria, 2011, p. 5), pondremos nuestro granito (nuestra letra) para ir cambiando la escuela y la sociedad. Concebir las formas del lenguaje como algo neutral equivale a pensar las formas de la cultura como algo neutral, que, como tal, no beneficia ni perjudica a nadie, que no encierra ningún interés. Preocupante, y absurdo. Pareciera que las niñas son tales mientras el niño se mantenga fuera de escena. Niñas,... hasta que un nińo diga lo contrario, parece decirnos la Real Academia al afirmar que "los alumnos es la única forma correcta de referirse a un grupo mixto, aunque el número de alumnas sea superior al de alumnos varones" (2016d).

Liliana Daunes dice algo tan sencillo como profundo: "una niña y un niño no son dos niños" (Longo, Bruno y Pomacusi, 2007, p. 194). Así es: una niña es una niña y cien niñas y un niño son cien niñas y un niño. No es cuestión de "desdoblamientos", como plantea la Real Academia; no desdoblamos nada, visibilizamos dos personas diferentes. Es necesario "nombrar la diferencia. No estamos duplicando el lenguaje. Duplicar sería hacer una copia igual a otra, y no es el caso" (Daunes, citada en Longo et al., 2007, p. 194).

\section{No eS UNA CUESTIÓN LINGÜísTICA}

Comenzábamos este trabajo con un ejemplo de párrafo justificativo del uso del lenguaje masculino. Era del Ministerio de Educación. En otros casos, el Ministerio dice: "a fin de marcar la presencia de ambos sexos, hemos optado por usar términos genéricos, en la medida de las posibilidades del lenguaje, y la forma masculina en su tradicional acepción" (Ministerio de Educación, 2009 , p. 2). Suscribimos estas palabras del Ministerio, salvo la última frase, la que dice que cuando las posibilidades del lenguaje no lo permitan optarán por "la forma masculina en su tradicional acepción". Pensamos que si iniciamos esta lucha contra todo tipo de dominación y en favor de las personas excluidas (más arriba hemos visto abundante información de la situación de discriminación que vive hoy la mujer) debemos hacerlo en el mayor número de frentes posibles. El lenguaje es uno de esos frentes, clave para quienes nos desempeñamos en el mundo educativo. El lenguaje puede estar al servicio de la exclusión, de la mentira (Eco, 1977), o de la equidad. Nosotras, en los casos en los que no de posibilidades el lenguaje, adoptaremos una postura "beligerante" y optaremos por la forma femenina en su revolucionaria acepción. 
Por lo tanto, cada vez que hacemos referencia a personas de ambos sexos como, maestras y maestros por ejemplo, para facilitar la lectura intentamos hacerlo con un término genérico. Cuando no podemos lo hacemos en el género propio de la palabra persona: en femenino. De esta forma quedan incluidas todas las personas.

Es una cuestión ideológica. En este sentido estamos de acuerdo con lo que dice la Real Academia de que el hacer visible el género femenino tiene su fundamento en razones extralingüísticas (RAE, 2016b); solo que la Real Academia no considera estas razones extras -políticas, sociales- válidas. Para nosotras, sin embargo, sí lo son. Es imprescindible tenerlas en cuenta. No priorizamos construir una gramática correcta-sumisa. Paulo Freire (1993, p. 62) dice que "la coherencia tiene que demostrarse también en el lenguaje (...) El lenguaje machista no es un problema gramatical sino ideológico (...), cambiar el lenguaje es parte del proceso de cambiar el mundo". Así es, amigo Freire. Y nosotras, como sońamos con un mundo diferente optamos por un lenguaje diferente.

\section{REFERENCIAS BIBLIOGRÁFICAS}

ABC (2014, 14 de abril). Las mujeres cobran un 16\% menos que los hombres en la Unión Europea. $A B C$. Recuperado de http://www.abc.es/economia/20140414/abci-mujeres-salarios-hombres-201404141424.html

Bruner, J. (1989). Acción pensamiento y lenguaje. Madrid: Alianza.

Camacho, G. (2014). La violencia de género contra las mujeres en el Ecuador. Quito, Ecuador: UNICEF.

Eco, U. (1977). Tratado de semiótica general. Barcelona, España: Lumen.

El Mundo (2016, 1 de enero). El año termina con 57 mujeres asesinadas por violencia de género. El Mundo. Recuperado de http://www.elmundo.es/socie dad/2015/12/31/56852bc6268e3ebf238b456d.html

Flores, J. y Holzscheiter, A. [2008]. El poder del discurso y el discurso del poder. Universidad de Berlín. Recuperado de jaf.lenguasindigenas.mx/docs/el_ poder_y_el_discurso.pdf

Freire, P. (1993). Pedagogía de la esperanza. Madrid, España: Siglo XXI.

INEC (2012). Infoeconomía. Publicación 3, septiembre de 2012. Instituto Nacional de Estadísticas y Censos de Ecuador. Recuperado de http://www. ecuadorencifras.gob.ec/wp-content/descargas/Infoconomia/infoe3.pdf

Lamas, M. (2007). El género es cultura. V Campus Euroamericano de cooperación cultural. Portugal. Recuperado de https://www.wcl.american.edu/hracademy/Clase1-Lectura1_000.pdf.pdf 
Lledó, E. [2004]. Nombrar a las mujeres, describir la realidad. En Emakunde, Gobierno Vasco (ed.), Uso del lenguaje en el mundo laboral (pp. 373-412). Vitoria. Recuperado de http://cdd.emakumeak.org/ficheros/0000/0138/ Lenguaje_1.pdf

Lomas, C. (2011). El poder de las palabras y las palabras del poder. Textos de didáctica de la lenguay la literatura, (58), 9-21. Recuperado de http://textos.grao.com/ revistas/textos/058-competencia-comunicativa-y-educacion-democratica/ el-poder-de-las-palabras-y-las-palabras-del-poder

Longo, R., Bruno, A. y Pomacusi, M. (2007). Resistir con alegría: Diálogo con Liliana Daunes. En C. Korol (comp.), Hacia una pedagogía feminista: géneros y educación popular (pp. 187-199). Buenos Aires, Argentina: Editorial El Colectivo y América Libre.

Ministerio de Educación (2009). Actualización y fortalecimiento curricular de la educación básica 2010: $3^{\text {er }}$ año. Quito, Ecuador: Ministerio de Educación de Ecuador.

Ministerio de Educación (2014). Currículo de educación inicial. Quito, Ecuador: Ministerio de Educación de Ecuador.

Myers, D. (2006). Psicología. Madrid, España: Editorial Médica Panamericana.

Pérez de Lara, N. (2010). La experiencia de la diferencia en la investigación. En J. Contreras y N. Pérez de Lara (comps.). Investigar la experiencia educativa, 117-135. Madrid, España: Morata.

Público (2015, 14 de septiembre). La ONU denuncia que las mujeres ganan un $24 \%$ menos y solo ocupan el $25 \%$ de los cargos directivos. Público. Recuperado de http://www.publico.es/sociedad/onu-denuncia-mujeresganan-24.html

RAE(2005).Diccionariopanhispánicodedudas. RealAcademiaEspañola. Recuperado de http://lema.rae.es/dpd/srv/search?id=Tr5x8MFOuD6DVTIDBg

RAE (2014). Diccionario de la lengua española. Real Academia Española. (23a ed.). Madrid, España: Espasa-Calpe.

RAE (2016a). Académicos. Real Academia Española. Recuperado de http://www. rae.es/la-institucion/los-academicos/directores

RAE (2016b). Respuestas a las preguntas más frecuentes. Real Academia Española. Recuperado de http://www.rae.es/consultas/los-ciudadanosy-las-ciudadanas-los-ninos-y-las-ninas

Remacha, B. (2016, 5 de abril). La curiosa misoginia de la RAE. Eldiario.es. Recuperado de http://www.eldiario.es/cultura/RAE-institucion-tradicional mente-misogina_0_502200361.html 
Rosano, S. (2015). Análisis de la participación estudiantil en la vida escolar: Estudio de caso en dos escuelas ecuatorianas. Tesis doctoral, Universidad de Cantabria. Repositorio abierto de la Universidad de Cantabria. Recuperado de http:// repositorio.unican.es/xmlui/handle/10902/6167

Universidad de Cantabria (2011). Guía UC: comunicación en igualdad. Recuperado de http://www.upm.es/sfs/Rectorado/Gerencia/Igualdad/Lenguaje/Guia_\% 20uc_comunicacion_en_igualdad.pdf

Vila, Ignasi (2009). Lev S. Vigotsky: la psicología cultural y la construcción de la persona desde la educación. En J. Trilla (coord.), El legado pedagógico del siglo XX para la escuela del siglo XXI. Barcelona, España: Graó.

Vygotsky, Lev (1995). Pensamiento y lenguaje. Barcelona, España: Paidós. 